\title{
Control central de la temperatura corporal y sus alteraciones: fiebre, hipertermia e hipotermia
}

Central control of body temperature and its alterations: fever, hyperthermia and hypothermia.

Controle central da temperatura corporal e suas alterações: febre, hipertermia e hipotermia.

Yelson Alejandro Picón-Jaimes, MD., MSc (c). ${ }^{1}$ (D), Javier Esteban OrozcoChinome, MD. ${ }^{2} \mathbb{D}$, Jessica Molina-Franky, MD., PhD. ${ }^{3} \mathbb{D}$, Mabel Patricia FrankyRojas., TR., Esp., MSc. ${ }^{4}$ (D)

1. Médico, Diploma en Salud Pública, Candidato a Magíster en Bioética. Docente Universidad Pedro de Valdivia, Santiago, Chile.

2. Médico cirujano, Diploma en Dermatología. Médico de consulta externa Red Salud, Santiago, Chile.

3. Médico, Candidata a Doctora en Ciencias Biomédicas y Biológicas. Investigadora grupo de investigación HYGEA, Universidad de Boyacá, Tunja, Boyacá, Colombia.

4. Terapeuta Respiratoria, Especialista en Gerencia de Instituciones de Salud. Magíster en Educación. Docente programa de Terapia Respiratoria, Universidad de Boyacá, Tunja, Boyacá, Colombia.

Correspondencia. Yelson Alejandro Picón-Jaimes. Médico, Diploma en Salud Pública, Candidato a Magíster en Bioética. Santiago, Chile. Tel. +56 948991158. Dirección. Avenida Pocuro 2260, Providencia, Chile. Email. ypicon@unab.edu.co

Cómo citar: Picón-Jaimes YA, Orozco-Chinome JE, Molina-Franky J, Franky-Rojas MP. Control central de la temperatura corporal y sus alteraciones: fiebre, hipertermia e hipotermia. MedUNAB. 2020;23(1):118-130. doi:10.29375/01237047.3714

\section{INFORMACIÓN DEL ARTÍCULO:}

Artículo recibido: 16 de septiembre de 2019

Artículo aceptado: 09 de enero de 2020

DOI: https://doi.org/10.29375/01237047.3714

\section{RESUMEN}

Introducción. En mamíferos, el control de la temperatura corporal es vital. El estado de consciencia y control motor en humanos, ocurren a una temperatura de $37^{\circ} \mathrm{C}$ y las desviaciones pueden alterar las propiedades celulares, generando disfunciones fisiológicas. En especies como los roedores (su relación área de superficie/volumen 
facilita la pérdida de calor) mantienen temperaturas basales cercanas a los $30^{\circ} \mathrm{C}$. Distinto es con animales como los paquidermos, cuya temperatura es menor comparada con los humanos. El objetivo es identificar los aspectos fisiológicos de la termorregulación. Descripción de temas tratados. Revisión descriptiva de la literatura de artículos publicados en diferentes bases de datos. La termorregulación es la capacidad del cuerpo para establecer y mantener su temperatura, regulando producción y pérdida de calor para optimizar la eficiencia de procesos metabólicos. El protagonismo lo tiene el sistema nervioso central y su control neurohormonal en múltiples niveles. El centro regulador térmico está en el hipotálamo anterior. Este recibe información de los receptores de grandes vasos, vísceras abdominales, médula espinal y de la sangre que perfunde el hipotálamo. Cuando aumenta la temperatura central, el termorregulador activa fibras eferentes del sistema nervioso autónomo, provocando pérdida de calor por convección y evaporación. Ante el descenso de temperatura, la respuesta es disminuir la pérdida de calor (vasoconstricción y menor sudoración); además, incrementar la producción de calor, intensificando la actividad muscular. Conclusión. La termorregulación es liderada por el hipotálamo, quien regula aumento y disminución de la temperatura respondiendo a las necesidades del organismo para llegar a la homeostasis y compensación, enfrentando las alteraciones de la temperatura ambiental

Palabras claves:

Temperatura Corporal; Termorreceptores; Hipotermia; Fiebre; Hipertermia.

\section{ABSTRACT}

Introduction. In mammals, controlling body temperature is vital. Consciousness and motor control in humans occur at a temperature of $37^{\circ} \mathrm{C}$ and any deviation can alter the cellular properties, generating physiological dysfunctions. In species such as rodents (their surface area/volume ratio facilitates heat loss) they maintain basal temperatures close to $30^{\circ} \mathrm{C}$. This is different with animals such as pachyderms, whose temperature is lower compared to humans. This article aims to Identify the physiological aspects of thermoregulation. Topics. Descriptive literature review of articles published in different databases. Thermoregulation is the body's ability to establish and maintain its temperature, regulating heat production and loss to optimize the efficiency of metabolic processes. The main actor in this process is the central nervous system and its neuro-hormonal control on multiple levels. The thermal regulating center is located in the anterior hypothalamus. It receives information from the receptors of large vessels, abdominal viscera, spinal cord and the blood that perfuses the hypothalamus. When the core temperature increases, the thermoregulator activates efferent fibers of the autonomic nervous system, causing heat loss by convection and evaporation. When the temperature drops, the response is to decrease heat loss (vasoconstriction and less sweating); in addition, increase heat production by intensifying muscle activity. Conclusion. Thermoregulation is led by the hypothalamus, which regulates temperature increase and decrease by responding to the organism's need to reach homeostasis and compensation, facing the alterations of the ambient temperature.

Keywords:

Body Temperature; Thermoreceptors; Hypothermia; Fever; Hyperthermia.

\section{RESUMO}

Introdução. Nos mamíferos, o controle da temperatura corporal é vital. O estado de consciência e controle motor em humanos ocorre a uma temperatura de $37^{\circ} \mathrm{C}$ e os desvios podem alterar as propriedades celulares, gerando disfunções fisiológicas. Espécies como os roedores (a sua relação superfície/volume, facilita a perda de calor), mantêm a temperatura basal próxima de $30{ }^{\circ} \mathrm{C}$. É diferente em animais como paquidermes, cuja temperatura é mais baixa em comparação aos humanos. Objetivo. Identificar os aspectos fisiológicos da termorregulação. Desenvolvimento. Revisão descritiva da literatura de artigos publicados em diferentes bases de dados. A termorregulação é a capacidade do corpo de estabelecer e manter sua temperatura, 
regulando a produção e a perda de calor para otimizar a eficiência dos processos metabólicos. O sistema nervoso central tem o papel principal, assim como seu controle neuro-hormonal em múltiplos níveis. O centro de regulação térmica está no hipotálamo anterior, que recebe informações dos receptores de grandes vasos, vísceras abdominais, medula espinhal e do sangue distribuído pelo hipotálamo. Quando a temperatura central aumenta, o termorregulador ativa fibras eferentes do sistema nervoso autônomo, causando perda de calor por convecção e evaporação. Dada a diminuição da temperatura, a resposta é reduzir a perda de calor (vasoconstrição e menos transpiração), além de aumentar a produção de calor, intensificando a atividade muscular. Conclusão. A termorregulação é liderada pelo hipotálamo, que regula o aumento e a diminuição da temperatura, respondendo às necessidades do organismo de atingir a homeostase e a compensação, enfrentando mudanças na temperatura ambiente.

Palavras-chave:

Temperatura Corporal; Termorreceptores; Hipotermia; Febre; Hipertermia.

\section{Introduction}

La termorregulación es un proceso natural del cuerpo que consiste en la activación de mecanismos centrales y periféricos para mantener la homeostasis corporal y las funciones vitales constantes (1-3). Su importancia está relacionada con la estabilidad de los procesos cardiovasculares, respiratorios, renales, endocrinos, nerviosos y el funcionamiento de los músculos, además, presenta vías complejas que permiten un vínculo estrecho entre estímulo y respuesta donde se involucran las vías aferentes y eferentes $(4,5)$.

Comprende mecanismos controlados por el hipotálamo, funciona a través de un sistema de retroalimentación que permite el aumento o disminución de la temperatura como respuesta a las condiciones ambientales gracias a la información que recibe de los diferentes sensores térmicos (neuronas especializadas sensibles al frío y al calor), los cuales poseen la capacidad de detectar las variaciones en la temperatura interna del organismo y compararla con la del medio. Los receptores se encuentran localizados en la piel, en la médula espinal, en los órganos internos y especialmente, en la región posterior del hipotálamo, manteniendo como valor de referencia $37^{\circ} \mathrm{C}(6-8)$.

La regulación de la temperatura corporal juega un papel fundamental en el individuo, debido a que el humano responde a modulaciones en el nivel de temperatura con respuestas voluntarias (cambiar de actividad física, reacciones de protección, abrigo, entre otros) $(9,10)$. Con la edad, la efectividad de la termorregulación disminuye, producto del deterioro sensorial; en el anciano hay disminución del metabolismo basal, pérdida de masa muscular y tono vascular, lo que lleva a un mayor peligro de hipotermia (11-13). Existen mecanismos de regulación que llevan a la pérdida de calor, dentro de los cuales se encuentran: la radiación, la convección, la conducción y la evaporación (12).

La radiación hace referencia al movimiento de energía en forma de rayos infrarrojos entre el medio externo y el cuerpo. La magnitud de la radiación emitida por el cuerpo se rige a favor de un gradiente de concentración que se establece hacia objetos distantes más fríos presentes en el ambiente, los cuales no necesariamente están en contacto directo con la superficie corporal. Se calcula que aproximadamente este mecanismo representa el $60 \%$ de la pérdida de calor corporal $(9,13)$.

La convección consiste en la transferencia de calor desde el cuerpo hacia partículas libres en el ambiente como las de aire o agua. Estas partículas ganan energía calórica al interactuar con la superficie corporal y, posteriormente, cuando se separan son reemplazadas por otras partículas frías que, a su vez, ganan energía; de esa forma el ciclo se repite, generando pérdida de calor. Este mecanismo es responsable de hasta el 12\% de la pérdida de calor $(2,4-6)$.

La conducción corresponde a la pérdida de calor corporal que se da cuando objetos entran en contacto directo con la superficie del cuerpo. Este proceso genera que la temperatura del objeto y la del cuerpo se equilibren, y una vez esto sucede, evita que el sujeto continúe perdiendo energía calórica. Por este mecanismo, se puede llegar a una pérdida de calor corporal del 3\%. Sin embargo, si el objeto que entra en contacto directo es agua fría, la pérdida de calor por esta reacción es, al menos, 100 veces mayor que por el aire frío $(3,10)$.

La evaporación aumenta la pérdida de calor de la superficie del cuerpo y es una estrategia termorreguladora crítica en temperaturas ambiente que exceden la temperatura central; el sudor enfría la piel y de forma secundaria los órganos internos. El mantenimiento de 
la sudoración como mecanismo compensador requiere el aporte de líquidos e iones, fundamentalmente cloro y sodio; en este mecanismo, es determinante la humedad ambiental, dado que la presencia y elevación de ésta, evita la evaporación del sudor y, por tanto, el descenso de temperatura $(14,15)$.

El presente artículo describe la termorregulación, haciendo mayor énfasis en las principales alteraciones, tales como la fiebre, hipertermia e hipotermia, partiendo de conceptos básicos como las respuestas fisiológicas mediadas por diversos factores internos y externos, así como la adaptación mediante vías nerviosas.

\section{Revisión de temas tratados}

Se realizó una revisión descriptiva de los mecanismos del control de la temperatura, se incluyeron artículos de revisión, artículos originales, revisiones sistemáticas y revisiones de casos publicados en bases de datos electrónicas como: Pubmed central y bibliotecas virtuales como Scielo, Medline, Ovid y Proquest. Se seleccionaron las palabras clave para la búsqueda: termorregulación, fisiología, humanos, homeostasis. Fueron escogidos todos los artículos que estuvieran disponibles en texto completo libre, publicados entre 2006 y 2018, escritos en idioma inglés o español. Se excluyeron textos duplicados, o que no se contaba con su versión completa. Posteriormente, se aplicó la estrategia de resumen analítico de investigación, con la cual se catalogaron los artículos más recientes que hacían referencia al tema de interés, obteniéndose 52 artículos en total y un libro, con los cuales se desarrolló el manuscrito (16).

\section{Aspectos fisiológicos de la termorregulación}

La defensa de la temperatura del cuerpo contra los fenómenos térmicos del ambiente es una de las funciones fundamentales homeostáticas dirigidas por el sistema nervioso. Los termorreceptores de la piel envían información térmica a los núcleos parabraquiales (NPB) de la médula para alcanzar el principal integrador de termorregulación, localizado en el Área Preóptica del Hipotálamo (APOH) (17-20) (figura 1).

Figura 1. Mecanismos de control de la temperatura

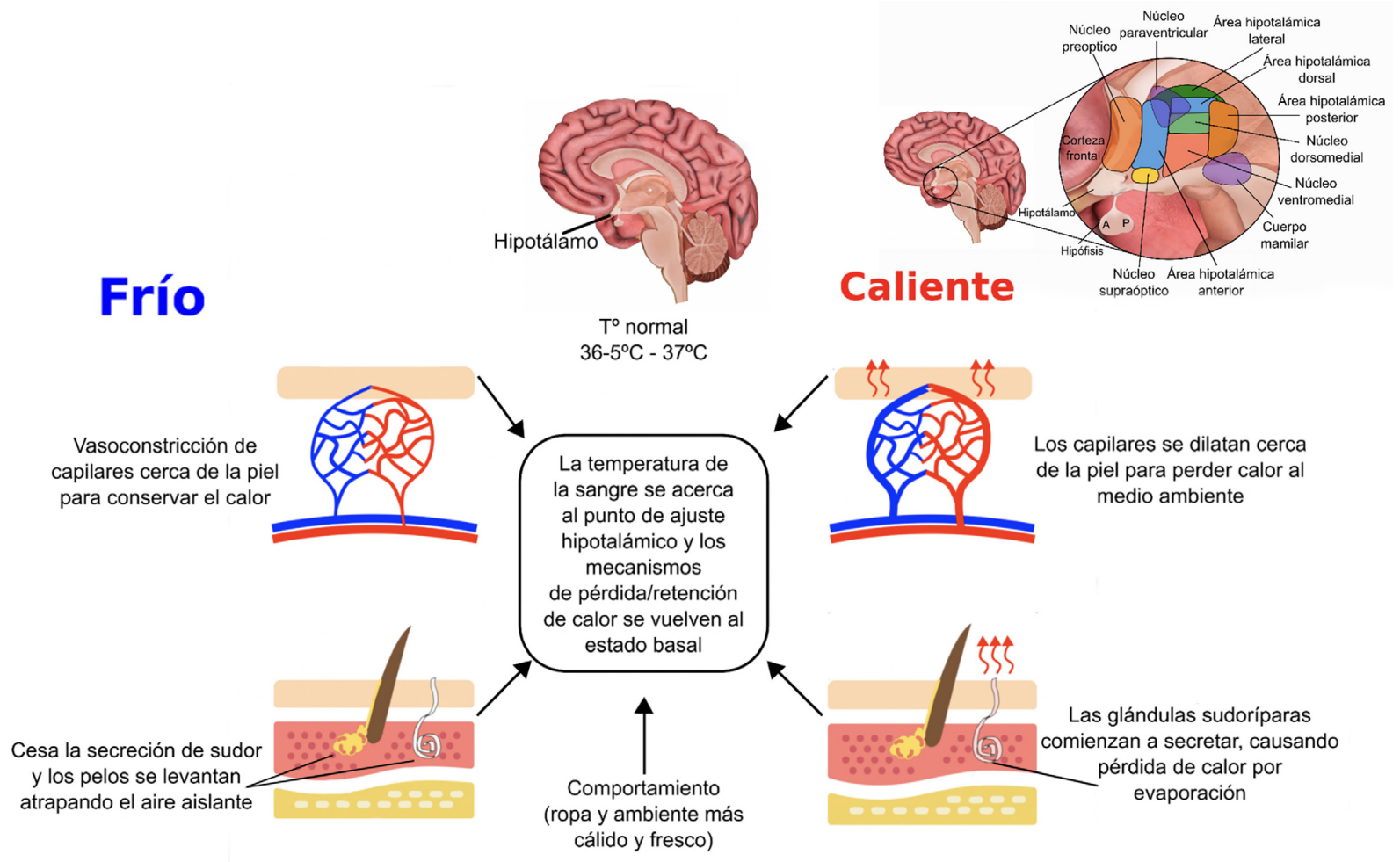

Fuente. Elaboración de los autores 
El APOH es el centro termorregulador encargado de proveer señales de orden descendente a los efectores periféricos para recordar conductas, autonómicas, somáticas y respuestas hormonales que contrarrestan los cambios en la temperatura ambiental, evitando que afecten a la temperatura corporal central. Las neuronas de comando de termorregulación en el APOH necesitan recibir la señalización directa de la información de la temperatura ambiental mediante termorreceptores de la piel, Corpúsculos de Ruffini (calor), Krause (frío), y a través de las astas dorsales de la médula espinal y del nervio trigémino (21-24).

La vía central más conocida para la señalización somatosensorial térmica cutánea, es la vía espinotalámicocortical. En dicha vía, las señales de termorreceptores de la piel se transfieren a través de una proyección directa del asta dorsal al tálamo y, luego son transmitidos a la corteza somatosensorial primaria, donde se hace consciente la temperatura del cuerpo (25-28).

\section{Mecanismos de la termorregulación}

Los mecanismos de la termorregulación se pueden clasificar en aquellos de tipo reflejo o autonómico y de conducta o voluntarios. En los mecanismos tipo reflejo, el sistema nervioso autónomo activa una serie de respuestas termorreguladoras ante los cambios de la temperatura corporal de forma automática y sin intervención de la voluntad del sujeto. En los mecanismos de conducta, el sujeto es consciente de tomar ciertas decisiones cuando siente que pierde su confort térmico, aunque su temperatura corporal no necesariamente varía. Ambos tipos de regulación contribuyen a conservar la homeostasis térmica (29) (figura 2).

Figura 2. Mecanismo de control voluntario y reflejo de la temperatura

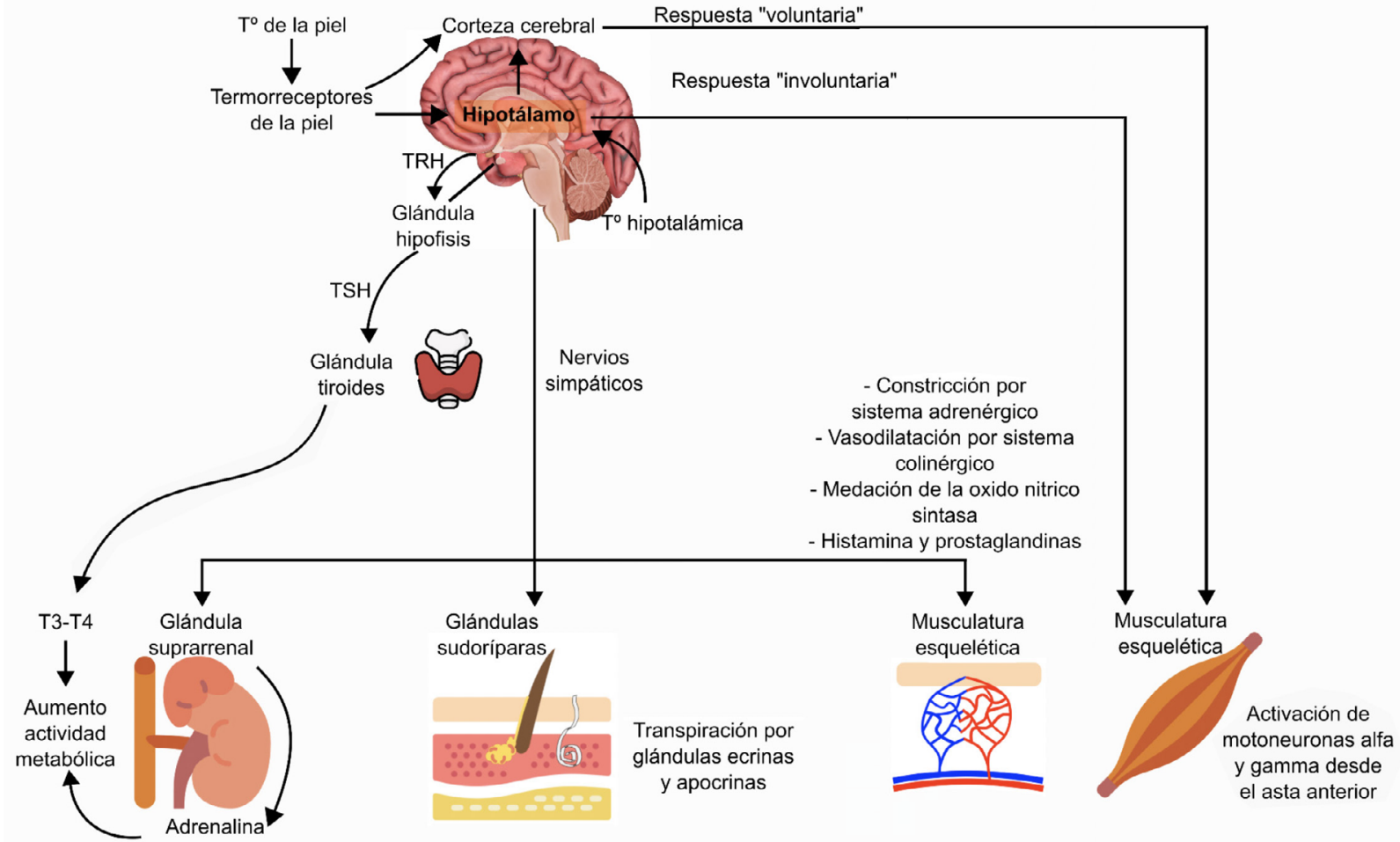

Fuente. Elaboración de los autores

\section{Termorregulación refleja}

El arco reflejo tiene tres grandes modalidades que permiten regular diferentes variables fisiológicas: Retrocontrol negativo, retrocontrol positivo $y$ retrocontrol anticipado. Este concepto habitual establece una forma básica de organizar la información fisiológica hasta hoy, especialmente para explicar mecanismos autonómicos, como es el caso de la termorregulación refleja (29).

La termorregulación refleja produce dos grandes fenómenos: la Respuesta Termorreguladora al Frío (RTF) y Respuesta Termorreguladora al Calor (RTC). 
La RTF es una combinación integrada de respuestas automáticas que incluye: termogénesis simpática en el tejido adiposo pardo, termogénesis por escalofrío en el músculo esquelético, incremento en la producción de dióxido de carbono espirado por aumento de la actividad metabólica y taquicardia. Por su parte, la RTC incluye vasodilatación cutánea, taquicardia y sudoración $(2,3)$.

\section{Vía aferente y vía espinal-parabraquial autonómica de la termorregulación refleja}

En esta vía, los termorreceptores constituyen neuronas aferentes primarias, cuyos somas se ubican en los ganglios de la raíz dorsal y núcleo espinal del nervio trigémino. Estos transmiten información térmica, periférica y somática del cuerpo y la cara, respectivamente. En el caso del cuerpo, el axón de estas neuronas hace sinapsis con neuronas aferentes secundarias en la lámina "I" de la asta dorsal de la médula espinal $(3,8)$.

Las neuronas aferentes secundarias de la lámina I de la asta dorsal, se dividen en poblaciones separadas que incluyen: neuronas nociceptivas específicas activadas por estímulos mecánicos y térmicos, neuronas nociceptivas polimodales activadas ante estímulos mecánicos, estímulos térmicos de frío y calor, y neuronas termorreceptivas específicas que se clasifican, simultáneamente, en "frías" y "calientes"; estas se activan cuando la piel es expuesta a frío y calor inocuo, respectivamente (3).

En la vía aferente del cuerpo, las neuronas frías de la lámina I de la asta dorsal, se proyectan hacia el núcleo parabraquial lateral del mesencéfalo, específicamente a los subnúcleos externo lateral y central. Por su parte las neuronas calientes se proyectan al subnúcleo dorsal del núcleo parabraquial lateral. Dado que las neuronas frías y calientes de la lámina "I" secretan glutamato, su activación estimula a las neuronas del mesencéfalo $(30,31)$.

\section{Comparaciones entre termorregulación de conducta y termorregulación refleja}

La termorregulación refleja es activada cuando se presentan estímulos térmicos que modifican la temperatura corporal y no requiere de percepción térmica consciente de los individuos (de hecho, como depende del hipotálamo, funciona por igual en individuos sanos conscientes e individuos dormidos o, incluso, inconscientes debido a daño en la corteza cerebral). La termorregulación de conducta se puede dar, igualmente, ante la presencia de estímulos térmicos o antes de que estos se presenten, ya que el individuo puede hacer uso de materiales externos que evitan dichos estímulos; para su activación, es indispensable la percepción consciente del individuo de perder el confort térmico, o de saber que lo va a perder $\mathrm{y}$, no necesariamente, requiere de alteraciones de la temperatura corporal (depende altamente de la corteza cerebral y necesariamente requiere que el sujeto esté consciente) $(8,30)$.

\section{Órganos involucrados en el incremento y detrimento de la temperatura}

Múltiples órganos y sistemas del cuerpo se ven afectados cuando la termorregulación no funciona correctamente.

\section{Vasos sanguíneos de la piel}

En respuesta al aumento o disminución de la temperatura ambiente o interna, el flujo sanguíneo de la piel es modificado a través de la vasodilatación simpática y mecanismos de vasoconstricción, respectivamente. El calor se disipa del cuerpo cuando la sangre se lleva muy cerca de la superficie de la piel, esto se logra a través de la vasodilatación. $(17,19)$.

La piel con folículos pilosos está inervada por el sistema noradrenérgico que produce vasoconstricción y por nervios colinérgicos que producen vasodilatación, mientras que la piel sin folículos, presente en las palmas, las plantas y los labios, es inervada únicamente por fibras nerviosas vasoconstrictoras que, en normotermia, mantienen un tono basal de vasoconstricción. En la piel con folículos pilosos, la principal respuesta al calor es aumentar el flujo sanguíneo cutáneo a través de la vasodilatación pasiva de vasos sanguíneos y mediante el bloqueo de la actividad nerviosa simpática $(17,23)$.

La presencia de numerosas anastomosis arteriovenosas en la piel glabra, puede conducir a grandes cambios en el flujo sanguíneo a estas regiones. Por ejemplo, en el calor, las anastomosis arteriovenosas se abren y la sangre fluye directamente de arterias a venas, evitando la alta resistencia de las arteriolas y las asas capilares. En la piel sin folículos pilosos, si la pérdida de calor por convección resultante de la relajación del tono vasoconstrictor es insuficiente para refrescar el núcleo, entonces puede ocurrir un aumento adicional en el flujo sanguíneo de la vasodilatación activa de la piel, aumentando así la pérdida de calor por convección. Esta 
vasodilatación activa es al menos, en parte, consecuencia de la respuesta a la liberación de acetilcolina y otros transmisores de los nervios colinérgicos simpáticos con la capacidad de aumentar el flujo sanguíneo cutáneo desde $300 \mathrm{ml} / \mathrm{min}$ hasta $8 \mathrm{~L} / \mathrm{min}(17,27)$.

La acetilcolina regula las respuestas vasodilatadoras activas frente al aumento de la temperatura corporal, sin embargo, se plantean otros cotransmisores responsables de esta respuesta, tales como: el péptido intestinal vasoactivo, sustancia $\mathrm{P}$, histamina, prostaglandinas y Receptor de Potencial Transitorio V1 (RPTV1). Otra hipótesis sugiere que los nervios colinérgicos, que controlan la sudoración, también producen vasodilatación activa al mismo tiempo. Por otra parte, es la enzima óxido nítrico sintasa (ONS) la responsable de producir óxido nítrico y, por tanto, ocasionar vasodilatación. Luego, si se inhibe dicha enzima, no puede haber óxido nítrico y no habrá vasodilatación por este mecanismo $(17,26)$.

Por último, frente a la disminución de la temperatura, los vasos sanguíneos presentan vasoconstricción simpática, la sangre se aleja de la superficie de la piel a través de las venas más profundas conservando así el calor. Otros cotransmisores liberados por el sistema simpático son el ATP y neuropéptido. Este último ha mostrado contribuir significativamente a la vasoconstricción del tono vascular cutáneo en respuesta al frío $(17,24)$.

\section{Glándulas sudoríparas}

El enfriamiento por transpiración es el único mecanismo de pérdida de calor cuando la temperatura ambiente excede la temperatura corporal. La exposición a un ambiente caluroso o al ejercicio, eleva las temperaturas del tronco y la piel, ambos contribuyen al aumento de la tasa de sudoración $(17,24)$.

El sudor es liberado por las glándulas sudoríparas (ecrinas y apocrinas) que se distribuyen en un gran número (de 1.6 a 4 millones) en la superficie del cuerpo. Están distribuidas de tal forma que, donde hay glándulas ecrinas (glándulas sudoríparas simples) no existe presencia de apocrinas $(14,17)$.

La evaporación del sudor permite que el calor se transfiera al medio ambiente como vapor de agua a través de las vías respiratorias y la superficie de la piel. El principal factor limitante en la capacidad de un ser humano para mantener la temperatura del cuerpo frente a un desafío térmico es la disponibilidad de agua para la producción de sudor (15).

\section{Tejido adiposo pardo (TAP)}

Se especializa en el proceso de termogénesis no hibernal, donde el metabolismo oxidativo se desacopla de la producción de ATP y, en el proceso, gasta energía. Este tejido es termogénico al aumentar la tasa metabólica. Este tejido es termogénico, aumenta la tasa metabólica $\mathrm{y}$, de ese modo, genera calor. Hasta hace poco, se creía que solo era importante en pequeños mamíferos y neonatos, sin embargo, hay evidencia de la activación del TAP en humanos adultos, aunque se ha visto que disminuye su proporción al aumentar la masa muscular (17).

La activación del sistema nervioso simpático por parte de estímulos periféricos y termorreceptores centrales pueden estimular la termogénesis en el TAP. La liberación de catecolaminas en los receptores $\beta 3$ adrenérgicos pueden impulsar la acción de las proteínas de desacoplamiento en la membrana mitocondrial interna (UCP-1, UCP-2, UCP-3S y UCP-3L), especialmente la UCP-1, la cual se ha visto mayormente relacionada a la termogénesis. Esta proteína UCP-1 también es conocida como termogenina, permite el paso de hidrogeniones a través de la membrana mitocondrial interna sin producción de ATP. Es sabido que la actividad del sistema nervioso simpático se incrementa con el frío, lo que conlleva al aumento de la termogénesis $(17,21)$.

Igualmente, cabe mencionar que la termogénesis en el TAP puede ser modulada por un número de factores no térmicos, incluyendo hipoxia, infección, hipoglucemia, y estrés psicológico (31).

\section{Músculo esquelético}

El músculo esquelético es el último sistema involucrado en la respuesta termorreguladora al frío, especialmente si los otros mecanismos no logran compensar el descenso de la temperatura. La activación de este efector está dada por la acción sobre las motoneuronas alfa y gamma del asta anterior, -de la médula espinalque producen contracciones musculares seriadas y repetitivas conocidas como tremor, aumentando la generación de calor por aumento de la actividad celular muscular $(16,31)$.

\section{Sistema endocrino}

Uno de los principales mecanismos hormonales para la regulación de la temperatura se da cuando la glándula tiroidea secreta tiroxina, que se transforma 
en triyodotironina, la cual se encarga de regular la temperatura corporal al aumentar el metabolismo celular (20).

El hipotálamo detecta el descenso de temperatura y secreta el factor liberador de tirotrofina, que estimula a la adenohipófisis para que produzca y libere tirotrofina que, a su vez, genera en la tiroides la secreción de tiroxina. Esta hormona, como se explicó anteriormente, se transforma a su forma activa, actuando a nivel celular y provocando un aumento metabólico que produce energía en forma de calor. Contrariamente, el aumento de la temperatura corporal detiene este proceso $(18,31)$.

Otra hormona importante en el control de la temperatura corporal es la vasopresina u hormona antidiurética. Se encarga del control de los niveles de agua corporal, por tanto, durante un episodio que requiera de enfriamiento a través del mecanismo de sudor, sus niveles aumentarán evitando la pérdida de líquido por la vía renal y permitiendo así, de forma indirecta, que más líquido se elimine por transpiración $(27,30)$.

Por consiguiente, se considera que la termorregulación es un proceso que involucra múltiples sistemas corporales y no se trata simplemente de un mecanismo compensatorio, sino que está implícito en la cotidianidad de las funciones hormonales, neurológicas, respiratorias, cardiovasculares $\mathrm{y}$, en general, depende de la homeostasis de cada uno de estos sistemas.

\section{Alteraciones de la termorregulación}

En ocasiones, el control central de la temperatura sufre variaciones que hace que se presenten ciertas condiciones que se detallan a continuación:

\section{Hipotermia}

Es la disminución anormal de la temperatura corporal. La Organización Mundial de la Salud (OMS) la define como una temperatura rectal inferior a $35.5^{\circ} \mathrm{C}$ o una temperatura axilar inferior a $35.0^{\circ} \mathrm{C}(32)$. Se presenta cuando aumentan las pérdidas de calor o disminuye su producción con baja de la temperatura corporal. Los descensos térmicos patológicos, independientes de las condiciones externas, pueden ser causados por disminución en la producción de calor, en la excitación del centro de frío, en la disminución de la actividad en el centro de calor (como también después de la administración de antipiréticos tipo antiinflamatorios tipo no esteroideos-AINES), en la parálisis de toda la regulación térmica central e igualmente por hacerse más lenta la irrigación sanguínea en los órganos productores de calor $(33,34)$.

La exposición prolongada al exterior con aire muy frío es un factor determinante en la pérdida de calor, sin embargo, es la exposición al agua fría, por su capacidad de conducción, el principal factor causante de estos eventos, en especial si no hay compensación por medio del metabolismo, el tono muscular y la vasoconstricción periférica. La hipotermia se revela con mucha frecuencia en hipotonía muscular, en el curso de la anestesia y la sedación (particularmente con barbitúricos, que son hipotermizantes), en la vasodilatación periférica, en el shock y en la reducción de la actividad metabólica en las fases terminales de muchas enfermedades.

En el shock, el descenso nocivo de la temperatura es percibido con facilidad y tiene importancia por ser, a menudo, un parámetro de mortalidad, especialmente cuando la curva térmica que desciende profundamente se cruza con la del pulso que asciende bruscamente. Ambos son resultado de una actividad simpática aumentada, ocasionando vasoconstricción periférica y taquicardia: una mediada por receptores alfa 1 y la otra por receptores beta $1(35,36)$. Si la temperatura corporal desciende por debajo de $30^{\circ} \mathrm{C}$ se producen profundas alteraciones funcionales y degenerativas en el hígado, miocardio y riñones que llevan rápidamente a la muerte (11).

\section{Hipertermia}

Es un trastorno de la regulación de la temperatura corporal que se caracteriza por una elevación de la temperatura central superior a $38.3^{\circ} \mathrm{C}(32-36)$. Independientemente de los factores etiológicos, ya sea por un exceso en la producción de calor, o por un defecto en la pérdida de este, se produce un aumento de la temperatura corporal que sobrepasa la capacidad de los mecanismos de termorregulación del organismo $(37,38)$. Puede presentarse con sudoración, sofoco, taquicardia, fatiga, mareo, dolor de cabeza y parestesias, progresando a hipotensión, síncope, confusión, delirio, convulsiones y coma. Los cambios en el estado mental y la temperatura central distinguen el golpe de calor potencialmente fatal del agotamiento por calor (11).

La Hipertermia maligna es una respuesta hipermetabólica que ocurre en un paciente genéticamente susceptible (mutaciones en el gen de la rianodina) que se expone a un estímulo gatillante, sin embargo, cualquier homo sapiens puede desarrollar este evento, particularmente 
si se expone a altas temperaturas externas o sufre deshidratación masiva durante una competencia. El estímulo desencadenante provoca la liberación masiva de calcio desde el retículo sarcoplásmico de la célula muscular.

El receptor de rianodina, que es una proteína que actúa como canal de calcio y funciona según el gradiente electroquímico del medio, es responsable de la salida masiva de calcio desde el retículo sarcoplásmico (su acción se contrapone a la bomba SERCA- sarco/ endoplasmic reticulum Ca- que invierte ATP para concentrar calcio al interior del retículo sarcoplásmico). El calcio liberado produce activación de las fibras de actina y miosina, generando contracción muscular, aumento del metabolismo, consumo de ATP y $\mathrm{O}_{2}$, producción de $\mathrm{CO}_{2} \mathrm{y}$ calor, acidosis láctica $\mathrm{y}$, finalmente, destrucción celular, con liberación de potasio y creatinkinasa (10). Todo lo anterior, se traduce en mioglobinuria, insuficiencia renal, alteraciones hemodinámicas, taquicardia e hipertensión arterial, arritmias y finalmente la muerte (39-41).

\section{Fiebre}

Constituye el trastorno más importante del equilibrio térmico y se presenta debido a una modificación funcional del centro termorregulador ubicado en el hipotálamo (41). Es necesario tener en cuenta que no es lo mismo hipertermia que fiebre. Mientras que las hipertermias, como las que resultan del ejercicio en un ambiente caluroso, surgen de una compensación incompleta del sistema termorregulador debido a una carga de calor impuesta; la fiebre es una elevación regulada de la temperatura central inducida por el propio sistema termorregulador central $(42,43)$.

\section{Causas y mecanismos del inicio de la fiebre}

La hipertermia febril se atribuye casi siempre a una respuesta inflamatoria mediada por linfocitos $\mathrm{TH} 1$, frente a la invasión del organismo por parte de microorganismos con potencial patogénico. Así, como a los casos acompañados de destrucción de tejidos con la consecuente producción de sustancias pirógenas. Cuando hay fiebre se presenta un aumento de la producción de calor, pero por sí sólo no explica la pirexia, ya que las alteraciones que ocurren son de una magnitud tal que podrían, fácilmente, ser compensadas en condiciones normales; simultáneamente, disminuyen las pérdidas de calor $\mathrm{y}$, en realidad, lo que explica es el aumento de temperatura del organismo. Por consiguiente, en los estados febriles, la temperatura del cuerpo se reorganiza a un nivel más elevado que el normal, como si el termostato hipotalámico estuviera fijado a una temperatura más alta. Esto explica por qué los pacientes sienten frío y se cubren con mantas o más ropa a pesar de tener una temperatura corporal elevada $(44,45)$.

Para entender el mecanismo inflamatorio de la fiebre se debe considerar el papel fundamental que cumple el sistema inmunitario, principalmente los macrófagos que, al fagocitar partículas exógenas, ya sean bacterias, virus o toxinas, producen citocinas, las cuales son péptidos de señalización que median las respuestas inmunitarias innatas y adaptativas $(25,42)$.

Una de las citocinas con mayor protagonismo durante este proceso es la interleucina $1 \alpha$ que actúa sobre el endotelio capilar del Órgano Vascular de la Lámina Terminal (OVLT), localizado en la pared del tercer ventrículo cerebral $(14,19,42)$. Se debe destacar que el OVLT se caracteriza por la falta de protección de la barrera hematoencefálica, permitiendo así que la interleucina $1 \alpha$ sea capaz de alcanzar el hipotálamo, desencadenando la modificación del termostato natural (42-46).

En consecuencia, este proceso que se lleva a cabo mediante la acción de la interleucina $1 \alpha$ en el OVLT, desencadena la liberación de prostaglandina E2, la cual actúa sobre los receptores EP1, EP2, EP3 y EP4 en el termostato hipotalámico y logra generar la regulación al alta; aumenta así lo que sería el parámetro eutérmico, engañando al termostato natural para que considere que debe mantener la temperatura corporal en niveles superiores $(11,42,46)$.

\section{Hipertermia inducida por fármacos}

La hipertermia maligna, también conocida como fiebre maligna o hipertermia de la anestesia, es un síndrome hipermetabólico que aparece en individuos genéticamente susceptibles a ciertos fármacos utilizados durante los procesos de anestesia. Este trastorno posee un patrón hereditario autosómico dominante, por consiguiente, una posibilidad de heredabilidad del $50 \%$ (47).

Lafisiopatogenia de esta entidad involucraunaregulación anormal y prolongada de los receptores que controlan el flujo de calcio, como es el caso del receptor tipo 1 de rianodina. Durante la despolarización celular ocurre 
un cambio estructural en el receptor sensible al voltaje de tipo L (dihidropiridina), se genera una liberación exagerada de calcio del retículo sarcoplásmico en el músculo estriado esquelético. La elevación sostenida de la concentración de calcio mioplasmático desencadena un fenómeno de contracción permanente, seguido de liberación de energía en forma de calor y aceleración de la función mitocondrial, finalmente, se traduce en aumento del consumo de oxígeno y mayor producción de dióxido de carbono.

Si no se corrige de forma temprana el agotamiento del metabolismo aerobio, es inminente un aumento en el metabolismo anaerobio, llevando al cuerpo a un estado de acidosis mixta (metabólica y respiratoria), con la consiguiente falla de las bombas de membrana consumidoras de energía como lo son la bomba $\mathrm{Ca}++-$ adenosina trifosfatasa (ATPasa), Na $+/ \mathrm{K}+$ - ATPasa, entre otras $(15,19,47)$.

La hipertermia maligna es principalmente una patología del músculo esquelético, aunque se reconoce la interacción de los altos niveles de calcio intracelular en otros tejidos, por ejemplo, el corazón, cerebro, etc (46).

\section{Termogénesis}

La termogénesis, producción de energía térmica, es un componente fundamental en el proceso homeostático para mantener la temperatura del cuerpo durante el desafío de baja temperatura ambiental y juega un papel esencial en la elevación de la temperatura corporal durante la respuesta febril a la infección. Las principales fuentes de producción de calor metabólico son: la oxidación mitocondrial en el tejido adiposo pardo, el aumento de la frecuencia cardíaca y temblores en el músculo esquelético, mediados por el centro motor primario o centro de la tiritona $(47,48)$.

\section{Termogénesis en el recién nacido}

Los recién nacidos, en especiales aquellos con bajo peso al nacer, son similares a los animales poiquilotermos, sus temperaturas caen a menos que los cuidadores intervengan para calentarlos. Sin la capacidad de generar calor, estos individuos pueden morir por bajas temperaturas corporales. La termogénesis no temblorosa es el método principal de producción de calor para el niño de hasta un año, sin embargo, los bebés con bajo peso al nacer tienen poca o ninguna habilidad para generar calor a través de la termogénesis no temblorosa debido a la inmadurez de sus sistemas.
La vasoconstricción periférica es uno de los primeros pasos en la conservación del calor en el tronco junto con la producción de calor de la termogénesis no temblorosa (49-53).

\section{Conclusión}

Los seres humanos poseen un sistema de adaptación lento a los cambios de temperatura comparado con los mamíferos de menor tamaño, presentándose un deterioro paulatino ante la disminución de las exigencias ambientales. El cuerpo utiliza mecanismos fisiológicos, como la termorregulación, controlados por el hipotálamo. Su función es comandar el aumento o disminución de la temperatura en respuesta a condiciones ambientales, utilizando la información recibida de sensores térmicos localizados en la piel, medula espinal y órganos internos, manteniendo un valor de referencia promedio en $\operatorname{los} 37^{\circ} \mathrm{C}$. Todo esto, con el fin de llegar a un estado de homeostasis en el organismo, tener un mecanismo de compensación para cualquier alteración en la temperatura puesto que, si no es controlado, puede traer alteraciones y llevar a estados extremos de hipotermia o hipertermia según sea el caso.

Enlaregulacióndelatemperatura sepresentanfenómenos en la pérdida de calor como la evaporación, con la cual se enfrían la piel y los tejidos. Dicho mecanismo requiere de líquido e iones para su mantenimiento y, como se explicó anteriormente, está regulado por factores como la acción de la hormona antidiurética. La hipertermia se caracteriza por una elevación de la temperatura central, facilitada por alteraciones en los canales de calcio al interior de las membranas celulares, de forma que la fiebre se expresa como una elevación anormal de la temperatura acompañada de diversos cambios fisiológicos coexistentes con gran número de enfermedades orgánicas.

Una vez finalizada esta revisión de tema, quedan abiertos interrogantes como los futuros usos de esta información en el control de los trastornos de la termorregulación, así como la invitación a investigadores a desarrollar nuevas moléculas y dispositivos para mediar en estos procesos de cara a lograr un mejor avance en el control de situaciones como la fiebre, hipertermia e hipotermia.

\section{Conflictos de intereses}

Los autores declaran que durante la construcción de este manuscrito no existieron conflictos de interés. 


\section{Referencias}

1. Flouris A, Schlader Z. Human behavioral thermoregulation during exercise in the heat. Scand $\mathbf{J}$ Med Sci Sports [Internet]. 2015 [citado 6 de feb. de 2020];25(S1):52-64. Recuperado a partir de: http:// doi.wiley.com/10.1111/sms. 12349

2. Morrison S. Central control of body temperature. F1000Research [Internet]. 2016. [citado 6 de feb. de 2020];5:1-10. Recuperado a partir de: http:// f1000research.com/articles/5-880/vl.

3. López A. Actualidad en termorregulación. Pensar en Movimiento. Revista Ciencias del Ejercicio y la Salud [Internet]. 2014 [citado 6 de feb. de 2020];12(2):1-36. Recuperado a partir de: http://revistas.ucr.ac.cr/index. php/pem/article/view/14918

4. Cheshire W. Thermoregulatory disorders and illness related to heat and cold stress. Autonomic Neuroscience: Basic and Clinical. [Internet]. 2016 [citado 6 de feb. de 20];196:91-104. Recuperado a partir de: http://linkinghub.elsevier.com/retrieve/pii/ $\underline{\text { S1566070216300017 }}$

5. Nakamura K. Central circuitries for body temperature regulation and fever. Am J Physiol Regul Comp Physiol [Internet]. 2011 [citado 6 de feb. de 2020];301(5):1207-28. Recuperado a partir de: $\quad$ http://ajpregu.physiology.org/cgi/doi/10.1152/ ajpregu.00109.2011

6. Yang $\mathrm{X}$, Ruan HB. Neuronal Control of Adaptive Thermogenesis. Front Endocrinol (Lausanne) [Internet]. 2015 [citado 6 de feb. de 2020];6(September):149. Recuperado a partir de: $\quad$ http://journal.frontiersin.org/Article/10.3389/ fendo.2015.00149/abstract

7. Sund-Levander M, Grodzinsky E. Assessment of body temperature measurement options. Br J Nurs [Internet]. 2013 [citado 6 de feb. de 2020];22(16):942-50. Recuperado a partir de: http://www.magonlinelibrary. com/doi/abs/10.12968/bjon.2013.22.16.942

8. Flouris A. Functional architecture of behavioural thermoregulation. Eur J Appl Physiol [Internet]. 2011 [citado 6 de feb. de 2020];111(1):1-8. Recuperado a partir de: http://link.springer.com/10.1007/s00421010-1602-8

9. Fernández E, Vilavella C, Saúl E, Sánchez E, Balmont G. Golpe de calor. FMC - Form Médica Contin en Atención Primaria [Internet]. 2016 [cited 2016 Nov 8];23(6):318-27. Recuperado a partir de: http://www. fmc.es/es/golpe-calor/articulo/90455666/

10. Gómez A. Trastornos de la temperatura corporal. Offarm [Internet]. [cited 2017 Jan 1];48-53. Recuperado a partir de: http://www.elsevier.es/esrevista-offarm-4-articulo-trastornos-temperaturacorporal-13108301

11. Maté M, Robles J, BoscáA, Aguado F. Trastornos de la regulación de la temperatura. Medynet [Internet]. 2007 [citado 6 de feb. de 2020];127(5):1-66. Recuperado a partir de: http://www.medynet.com/usuarios/jraguilar/ Manual de urgencias y Emergencias/temp.pdf

12. Sessler D. The thermoregulation story. Anesthesiology [Internet]. 2013 [citado 6 de feb. de 2020];118(1):1816. Recuperado a partir de: http://anesthesiology. pubs.asahq.org/Article.aspx?doi=10.1097/ ALN.0b013e3182784df3

13. Hailes W, Cuddy J, Cochrane K, Ruby B. Thermoregulation During Extended Exercise in the Heat: Comparisons of Fluid Volume and Temperature. Wilderness Environ Med [Internet]. 2016 [citado 6 de feb. de 2020];27(3):386-92. Recuperado a partir de: http://linkinghub.elsevier.com/retrieve/pii/ $\underline{\text { S1080603216301053 }}$

14. Camacho D. Estrés térmico en trabajadores expuestos al área de fundición en una empresa metalmecánica. Cienc Trab [Internet]. 2013 [citado 6 de feb. de 2020];15(46):31-4. Recuperado a partir de: www. cienciaytrabajo.cl

15. Parker G, Brotchie $H$. Gender differences in depression. Int Rev Psychiatry [Internet]. 2010 [citado 6 de feb. de 2020];22(5):429-36. Recuperado a partir de: http://www.ncbi.nlm.nih.gov/pubmed/21047157

16. "Revista Virtual Universidad Católica del Norte". No.26, (febrero - mayo de 2009, Colombia), ISSN 0124-5821 - Indexada categoría C Publindex e incluida en Latindex

17. Tansey E, Johnson C. Recent advances in thermoregulation. Adv Physiol Educ [Internet]. 2015 [citado 6 de feb. de 2020];39(3):139-48. Recuperado a partir de: http://ajpadvan.physiology.org/lookup/ doi/10.1152/advan.00126.2014

18. Walloe L. Arterio-venous anastomoses in the human skin and their role in temperature control. Temperature [Internet]. 2016 [citado 6 de feb. de 2020];3(1):92103. Recuperado a partir de: http://www.ncbi.nlm.nih. gov/pubmed/27227081

19. Caron A, Richard D. Neuronal systems and circuits involved in the control of food intake and adaptive thermogenesis. Ann N Y Acad Sci [Internet]. 2016 [citado 6 de feb. de 2020];1-19. Available from: http:// doi.wiley.com/10.1111/nyas.13263

20. Passlick-Deetjen J, Bedenbender-Stoll E. Why thermosensing, A primer on thermoregulation. Nephrol Dial Transplant [Internet]. 2005 [citado 6 de feb. de 2020];20(9):1784-9. Recuperado a partir de: http://ndt.oxfordjournals.org/cgi/doi/10.1093/ndt/ gfh901

21. Hanna E, Tait P. Limitations to Thermoregulation and Acclimatization Challenge Human Adaptation to Global Warming. Int J Environ Res Public Health [Internet]. 2015 [citado 6 de feb. de 2020];12(7):8034 
74. Recuperado a partir de: http://www.mdpi. com/1660-4601/12/7/8034/

22. Lin G, Scott J. Investigations of the constitutive overexpression of CYP6D1 in the permethrin resistant LPR strain of house fly (Musca domestica). Pestic Biochem Physiol [Internet]. 2011[citado 6 de feb. de 2020];100(2):130-4. Recuperado a partir de: $\quad$ http://linkinghub.elsevier.com/retrieve/pii/ S0048357511000411

23. Benarroch E. Thermoregulation: Recent concepts and remaining questions. Neurology [Internet]. 2007 [citado 6 de feb. de 2020];69(12):1293-7. Recuperado a partir de: http://www.neurology.org/cgi/ doi/10.1212/01.wnl.0000275537.71623.8e

24. Morf J, Schibler U. Body temperature cycles: Gatekeepers of circadian clocks. Cell Cycle [Internet]. 2013 [citado 6 de feb. de 2020];12(4):539-40. Recuperado a partir de: http://www.tandfonline.com/ doi/abs/10.4161/cc.23670

25. Tupone D, Madden C, Morrison S. Autonomic regulation of brown adipose tissue thermogenesis in health and disease: potential clinical applications for altering BAT thermogenesis. Front Neurosci [Internet]. 2014 [citado 6 de feb. de 2020];8(8 FEB):1-14. Available from: http://journal.frontiersin. org/article/10.3389/fnins.2014.00014/abstract

26. Lomniczi A, Wright H, Ojeda S. Epigenetic regulation of female puberty. Front Neuroendocrinol [Internet]. 2015 [citado 6 de feb. de 2020];36:90-107. Recuperado a partir de: http://linkinghub.elsevier. com/retrieve/pii/S0091302214000752

27. Nakamura K, Morrison S. Central efferent pathways mediating skin cooling-evoked sympathetic thermogenesis in brown adipose tissue. AJP Regul Integr Comp Physiol [Internet]. 2012 [citado 6 de feb. de 2020];292(1):R127-36. Recuperado a partir de: http://ajpregu.physiology.org/cgi/doi/10.1152/ ajpregu.00427.2006

28. González-Alonso J. Human thermoregulation and the cardiovascular system. Exp Physiol [Internet]. 2012 [citado 6 de feb. de 2020];97(3):340-6. Recuperado a partir de: http://doi.wiley.com/10.1113/ expphysiol.2011.058701

29. Saito T, Sadoshima J. Molecular Mechanisms of Mitochondrial Autophagy/Mitophagy in the Heart. Circ Res [Internet]. 2015 [citado 6 de feb. de 2020];116(8):1477-90. Recuperado a partir de: http://circres.ahajournals.org/cgi/doi/10.1161/ CIRCRESAHA.116.303790

30. Nagashima K. Central Mechanisms for Thermoregulation in a Hot Environment. Ind Health [Internet]. 2006 [citado 6 de feb. de 2020];44(3):35967. Recuperado a partir de: http://joi.jlc.jst.go.jp/JST. JSTAGE/indhealth/44.359? from=CrossRef
31. Wang H, Siemens J. Ion channels in thermosensation, thermoregulation and metabolism. Temperature [Internet]. 2015 [citado 6 de feb. de 2020];2(2):17887. Recuperado a partir de: https://www.tandfonline. com/doi/full/10.1080/23328940.2015.1040604

32. World Health Organization (2019). Prevención y tratamiento de la hipotermia en niños con malnutrición grave. [online] Recuperado a partir de: https:// www.who.int/elena/titles/bbc/hypothermia_sam/es/ [Accessed 16 Dec. 2019]

33. Ramos G, Grünberg G. Evaluation of the incidence trembling in the recovery room postanesthtic Avaliação. Anest Analg Reanim [Internet]. 2016 [citado 6 de feb. de 2020];29(1):2. Recuperado a partir de: http://www.scielo.edu.uy/scielo.php?script=sci arttext\&pid=S1688-12732016000100002\&lang=pt

34. Cordero A, Cordero I. Temblores posanestésicos. Rev Cuba Anestesiol y Reanim [Internet]. 2016 [citado 8 de febrero de 2020];15(3):243-8. Recuperado a partir de: http://scielo.sld.cu/pdf/scar/v15n3/scar08316.pdf

35. Brito V, Galvão C, dos-Santos C. Factors associated to the development of hypothermia in the intraoperative period. Rev Lat Am Enfermagem [Internet]. 2009 [citado 8 de febrero de 2020];17(2):228-33. Recuperado a partir de: http://www.ncbi.nlm.nih.gov/ pubmed/19551277

36. Bissinger $\mathrm{R}$, Annibale D. Thermoregulation in Very Low-Birth-Weight Infants During the Golden Hour. Adv Neonatal Care [Internet]. 2010 [citado 8 de febrero de 2020];10(5):230-8. Recuperado a partir de: http://content.wkhealth.com/ linkback/openurl?sid=WKPTLP:landingpage\& an $=00149525-201010000-00004$

37. Sultan P, David A, Fernando R, Ackland G. Inflammation and Epidural-Related Maternal Fever. Anesth Analg [Internet]. 2016 [citado 8 de febrero de 2020];122(5):1546-53. Recuperado a partir de: http://content.wkhealth.com/linkback/openurl?sid=WKPTLP:landingpage\& $\mathrm{an}=00000539-201605000-00044$

38. Corvetto A, Heider M, Cavallieri B. Hipertermia maligna: ¿cómo estar preparados? Rev Chil cirugía [Internet]. 2013 [citado 8 de febrero de 2020];65(3):279-84. Recuperado a partir de: http://www.scielo.cl/scielo.php?script=sci arttext\&pid=S0718-40262013000300015\&lng=en\&n$\mathrm{rm}=$ iso\&tlng=en

39. González G, Ulloa B. Hipertermia maligna: adelantándose a las complicaciones. Rev Otorrinolaringol y cirugía cabeza y cuello [Internet]. 2006 [citado 8 de febrero de 2020];66(1):51-4. Recuperado a partir de: http://www.scielo.cl/scielo.php?script=sci arttext\&pid=S0718-48162006000100009\&lng=en\&n$\underline{\mathrm{rm}=\mathrm{iso} \& \mathrm{t} \operatorname{lng}=\mathrm{en}}$ 
40. Wang L, Zou Z, Hou C, Liu X, et Al. Score risk model for predicting severe fever with thrombocytopenia syndrome mortality. BMC Infect Dis [Internet]. 2017 [citado 8 de febrero de 2020];17(1):42. Recuperado a partir de: http://bmcinfectdis.biomedcentral.com/articles/10.1186/s12879-016-2111-0

41. Hurtado V, Sendoya F. Implicaciones en la salud de los trabajadores originadas por la exposición térmica en cultivos. Rev Colomb Salud Ocup. 2016;6(2):1-8. Doi: https://doi.org/10.18041/2322-634X/rcso.2.2016.4913

42. Horowitz H. Fever of Unknown Origin or Fever of Too Many Origins? N Engl J Med [Internet]. 2013 [citado 8 de febrero de 2020];368(3):197-9. Recuperado a partir de: $\underline{\mathrm{http}: / / w w w . n e j m . o r g / d o i / a b s / 10.1056 /}$ NEJMp1212725

43. Young P, Saxena M, Bellomo R, Freebairn R, Bellomo R, Hammonf N, et al. Acetaminophen for Fever in Critically Ill Patients with Suspected Infection. N Engl J Med [Internet]. 2015 [citado 8 de febrero de 2020];373(23):2215-24. Recuperado a partir de: http://www.nejm.org/doi/10.1056/NEJMoa1508375

44. Kuwaki T. Thermoregulation under pressure: a role for orexin neurons. Temperature [Internet]. 2015 [citado 8 de febrero de 2020];2(3):379-91. Recuperado a partir de: http://www.tandfonline.com/doi/full/10.108 $\underline{0}$ /23328940.2015.1066921

45. Zhen C, Xia Z, Ya Jun Z, Long L, Jian S, Gui-Ju C, et al. Accuracy of Infrared Tympanic Thermometry Used in the Diagnosis of Fever in Children: A Systematic Review and Meta-Analysis. Clin Pediatr (Phila) [Internet]. 2015 [citado 8 de febrero de 2020];54(2):114 26. Recuperado a partir de: http://cpj.sagepub.com/ cgi/doi/10.1177/0009922814545492

46. Smith C, Lee-Miller C, Dishop M, Cost C, et Al. Multicentric Castleman disease presenting with fever. J Pediatr [Internet]. 2014[cited 2016 Nov 3];165(6):12615. Recuperado a partir de: http://www.jpeds.com/article/S0022347614007896/fulltext

47. Morrison S, Nakamura K, Madden C. Central control of thermogenesis in mammals. Exp Physiol [Internet]. 2008 [citado 8 de febrero de 2020];93(7):773-97. Recuperado a partir de: http://doi.wiley.com/10.1113/ expphysiol.2007.041848

48. He Q, Johnston J, Zeitlinger J. ChIP-nexus enables improved detection of in vivo transcription factor binding footprints. Nat Biotechnol [Internet]. 2015 [citado 8 de febrero de 2020];33(4):395-401. Recuperado a partir de: http://www.nature.com/doifinder/10.1038/ nbt.3121

49. Sánchez J, Romero C, Muñoz L, Rivera R. El órgano adiposo, un arcoiris de regulación metabólica y endocrina. Rev Cuba Endocrinol. [Internet] 2016 [citado 8 de febrero de 2020];27(1):105-19. Recuperado a partir de: http://scielo.sld.cu/scielo.php?script=sci art- text\&pid $=$ S1561-29532016000100010

50. Scott H, Tyton T, Horswill C. Conducta ocupacional sedentaria y soluciones para aumentar la termogénesis no asociada al ejercicio. Rev ciencias del Ejerc y la salud. 2016;14(2):22-43. Doi: http://dx.doi. org/10.15517/pensarmov.v14i2.27496

51. Knobel-Dail R, Holditch-Davis D, Sloane B, Guenther D, Laurence M. Body temperature in premature infants during the first week of life: Exploration using infrared thermal imaging. Journal of Thermal Biology. 2017 ; 69: 118-123.

52. Kollmann-Camaiora A, Alsina E, Domínguez A, del-Blanco B, Yepes MJ, Guerrero JL, García. Clinical protocol for the management of malignant hyperthermia. Revista Española de Anestesiología y Reanimación. 2017; 64(1): 32-40. DOI: http://dx.doi. org/10.1016/j.redar.2016.06.004

53. Romanovsky A. Handbook of Clinical Neurology, Thermoregulation, From Basic Neuroscience to Clinical Neurology Part I, Volume 156. 1st ed. Oxford: Elsevier; 2018. 